\title{
5D black hole solution in Einstein-Yang-Mills-Gauss-Bonnet theory
}

\author{
S. Habib Mazharimousavi* and M. Halilsoy ${ }^{\dagger}$ \\ Department of Physics, Eastern Mediterranean University, G. Magusa, north Cyprus, via Mersin-10, Turkey \\ (Received 10 July 2007; published 4 October 2007)

\begin{abstract}
By adopting the 5D version of the Wu-Yang ansatz we present in closed form a black hole solution in the Einstein-Yang-Mills-Gauss-Bonnet theory. In the Einstein-Yang-Mills limit, we recover the 5D black hole solution already known.
\end{abstract}

DOI: 10.1103/PhysRevD.76.087501

PACS numbers: 04.50.+h, 04.20.Jb, 04.70.Bw

The $5 \mathrm{D}$ line element is chosen as

$$
d s^{2}=-f(r) d t^{2}+\frac{d r^{2}}{f(r)}+r^{2} d \Omega_{3}^{2},
$$

in which the $S^{3}$ line element will be expressed in the alternative form

$$
d \Omega_{3}^{2}=\frac{1}{4}\left(d \theta^{2}+d \phi^{2}+d \psi^{2}-2 \cos \theta d \phi d \psi\right),
$$

where

$$
0 \leq \theta \leq \pi, \quad 0 \leq \phi, \psi \leq 2 \pi .
$$

This form has a nearly Euclidean appearance with the advantage of admitting two explicit killing vectors $\partial_{\phi}$ and $\partial_{\psi}$. We introduce the Wu-Yang ansatz $[1,2]$ in $5 \mathrm{D}$ as

$$
\begin{aligned}
A^{i} & =\frac{Q}{r^{2}} \epsilon_{j k}^{i} x^{k} d x^{j}, \\
A^{i+3} & =\frac{Q}{r^{2}}\left(x^{i} d w-w d x^{i}\right) \quad(i, j, k=1,2,3),
\end{aligned}
$$

in which $r^{2}=x^{2}+y^{2}+z^{2}+w^{2}$ and $Q$ is the only nonzero gauge charge. The latter coordinates are expressed in terms of the Euler angles

$$
\begin{aligned}
& x+i y=r \sin \left(\frac{\theta}{2}\right) \exp i\left(\frac{\phi+\psi}{2}\right), \\
& z+i w=r \cos \left(\frac{\theta}{2}\right) \exp i\left(\frac{\phi-\psi}{2}\right) .
\end{aligned}
$$

It is observed that the reduction $S^{3} \rightarrow S^{2}$ amounts to taking $\phi=\psi$ and $\theta \rightarrow 2 \theta$. Although the isomorphism $S O(4) \equiv S O(3) \times S O(3)$ supports two independent sets of rotation matrices, this will not be our strategy here. Instead, we shall parametrize both groups in terms of the common Euler angles which implies mixing of the groups.

The gauge potential 1-forms in terms of the Euler angles have the following explicit form

\footnotetext{
*habib.mazhari@emu.edu.tr
}

†mustafa.halilsoy@emu.edu.tr

$$
\begin{aligned}
& A^{1}=\frac{Q}{4}(\cos \psi \sin \theta d \phi+\cos \phi \sin \theta d \psi+(\sin \phi+\sin \psi) d \theta), \\
& A^{2}=\frac{Q}{4}(\sin \psi \sin \theta d \phi+\sin \phi \sin \theta d \psi-(\cos \phi+\cos \psi) d \theta), \\
& A^{3}=-\frac{Q}{2} \sin ^{2}\left(\frac{\theta}{2}\right)(d \phi+d \psi), \\
& A^{4}=\frac{Q}{4}(\cos \psi \sin \theta d \phi-\cos \phi \sin \theta d \psi-(\sin \phi-\sin \psi) d \theta), \\
& A^{5}=\frac{Q}{4}(\sin \psi \sin \theta d \phi-\sin \phi \sin \theta d \psi+(\cos \phi-\cos \psi) d \theta), \\
& A^{6}=\frac{Q}{2} \cos ^{2}\left(\frac{\theta}{2}\right)(d \phi-d \psi) .
\end{aligned}
$$

The Yang-Mills (YM) field 2-forms are defined as follows:

$$
\begin{aligned}
& F^{1}=d A^{1}+\frac{1}{Q}\left(A^{2} \wedge A^{3}+A^{5} \wedge A^{6}\right) \\
& F^{2}=d A^{2}+\frac{1}{Q}\left(A^{3} \wedge A^{1}+A^{6} \wedge A^{4}\right) \\
& F^{3}=d A^{3}+\frac{1}{Q}\left(A^{1} \wedge A^{2}+A^{4} \wedge A^{5}\right) \\
& F^{4}=d A^{4}+\frac{1}{Q}\left(A^{2} \wedge A^{6}+A^{5} \wedge A^{3}\right) \\
& F^{5}=d A^{5}+\frac{1}{Q}\left(A^{6} \wedge A^{1}+A^{3} \wedge A^{4}\right) \\
& F^{6}=d A^{6}+\frac{1}{Q}\left(A^{4} \wedge A^{2}+A^{1} \wedge A^{5}\right)
\end{aligned}
$$

We note that our notation follows the standard exterior differential forms; namely, $d$ stands for the exterior derivative while $\wedge$ stands for the wedge product. The hodge star * in the sequel will be used to represent duality [3].

The integrability conditions

$d F^{1}+\frac{1}{Q}\left(A^{2} \wedge F^{3}-A^{3} \wedge F^{2}+A^{5} \wedge F^{6}-A^{6} \wedge F^{5}\right)=0$,

plus five other similar equations, are easily satisfied by using (6). The YM equations 


$$
\begin{aligned}
& d^{*} F^{1}+\frac{1}{Q}\left(A^{2} \wedge^{*} F^{3}-A^{3} \wedge^{*} F^{2}+A^{5} \wedge^{*} F^{6}-A^{6} \wedge^{*} F^{5}\right)=0, \\
& d^{*} F^{2}+\frac{1}{Q}\left(A^{3} \wedge^{*} F^{1}-A^{1} \wedge^{*} F^{3}+A^{6} \wedge^{*} F^{4}-A^{4} \wedge^{*} F^{6}\right)=0, \\
& d^{*} F^{3}+\frac{1}{Q}\left(A^{1} \wedge^{*} F^{2}-A^{2} \wedge^{*} F^{1}+A^{4} \wedge^{*} F^{5}-A^{5} \wedge^{*} F^{4}\right)=0, \\
& d^{*} F^{4}+\frac{1}{Q}\left(A^{2} \wedge^{*} F^{6}-A^{6} \wedge^{*} F^{2}+A^{5} \wedge^{*} F^{3}-A^{3} \wedge^{*} F^{5}\right)=0, \\
& d^{*} F^{5}+\frac{1}{Q}\left(A^{6} \wedge^{*} F^{1}-A^{1} \wedge^{*} F^{6}+A^{3} \wedge^{*} F^{4}-A^{4} \wedge^{*} F^{3}\right)=0, \\
& d^{*} F^{6}+\frac{1}{Q}\left(A^{4} \wedge^{*} F^{2}-A^{2} \wedge^{*} F^{4}+A^{1} \wedge^{*} F^{5}-A^{5} \wedge^{*} F^{1}\right)=0
\end{aligned}
$$

are all satisfied.

The energy-momentum tensor

$$
T_{\mu \nu}=2 F_{\mu}^{i \alpha} F_{\nu \alpha}^{i}-\frac{1}{2} g_{\mu \nu} F_{\alpha \beta}^{i} F^{i \alpha \beta},
$$

where $F_{\alpha \beta}^{i} F^{i \alpha \beta}=6 Q^{2} / r^{4}$ has the nonzero components

$$
\begin{aligned}
T_{t t} & =\frac{3 Q^{2} f(r)}{r^{4}}, \\
T_{r r} & =-\frac{3 Q^{2}}{r^{4} f(r)}, \\
T_{\theta \theta} & =T_{\phi \phi}=T_{\psi \psi}=\frac{Q^{2}}{4 r^{2}}, \\
T_{\phi \psi} & =T_{\psi \phi}=-\frac{Q^{2}}{4 r^{2}} \cos \theta .
\end{aligned}
$$

The Einstein-Yang-Mills-Gauss-Bonnet (EYMGB) equations [4],

$$
\begin{gathered}
G_{\mu \nu}-\alpha\left\{\frac{1}{2} g_{\mu \nu}\left(R_{\kappa \lambda \rho \sigma} R^{\kappa \lambda \rho \sigma}-4 R_{\rho \sigma} R^{\rho \sigma}+R^{2}\right)-2 R R_{\mu \nu}\right. \\
\left.\quad+4 R_{\mu}^{\lambda} R_{\nu}^{\lambda}+4 R^{\rho \sigma} R_{\mu \rho \nu \sigma}-R_{\mu}^{\rho \sigma \lambda} R_{\nu \rho \sigma \lambda}\right\}=T_{\mu \nu},
\end{gathered}
$$

reduce to the simple set of equations

$$
\begin{gathered}
2 r\left(-\frac{r^{2}}{4}+\alpha(f(r)-1)\right) f^{\prime}(r)+r^{2}(1-f(r))-Q^{2}=0, \\
r^{2}\left(\frac{r^{2}}{2}+2 \alpha(1-f(r))\right) f^{\prime \prime}(r)+2 r^{3} f^{\prime}(r)-2 \alpha r^{2} f^{\prime}(r)^{2} \\
+(f(r)-1) r^{2}-Q^{2}=0,
\end{gathered}
$$

in which a prime denotes derivative with respect to $r$.

This set admits the solution

$$
f(r)=1+\frac{r^{2}}{4 \alpha} \pm \sqrt{\left(\frac{r^{2}}{4 \alpha}\right)^{2}+\left(1+\frac{m}{2 \alpha}\right)+\frac{Q^{2} \ln (r)}{\alpha}},
$$

in which $m$ is the usual integration constant to be identified as mass. We notice that under the limit $\alpha \rightarrow 0$, and the $(-)$ sign [i.e., Einstein-Yang-Mills (EYM) limit], the solution reduces to [5]

$$
f(r)=1-\frac{m}{r^{2}}-\frac{2 Q^{2}}{r^{2}} \ln (r) .
$$

The difference of this solution from the 5D ReissnerNordstrom solution requires no comment.

By using (10) the energy density is

$$
\epsilon=-g^{t t} T_{t t}=\frac{3 Q^{2}}{r^{4}},
$$

whose integral diverges logarithmically as in the ReissnerNordstrom case. The surface gravity $\kappa$ defined by [6] [note that for our purpose we are choosing the ( - ) sign and $\alpha \geq$ 0 in (13)],

$$
\kappa^{2}=-\frac{1}{4} g^{t t} g^{i j} g_{t t, i} g_{t t, j}
$$

has the form

$$
\kappa=\left|\frac{1}{2} f^{\prime}\left(r_{+}\right)\right|=\left|\frac{r}{4 \alpha}-\left(\frac{r_{+}^{3}}{4 \alpha^{2}}+\frac{Q^{2}}{r_{+} \alpha}\right) \frac{1}{\sqrt{\Delta}}\right|,
$$

where

$$
\Delta=\frac{r_{+}^{4}}{\alpha^{2}}+16+\frac{8 m}{\alpha}+\frac{16 Q^{2} \ln \left(r_{+}\right)}{\alpha}
$$

and $r_{+}$is the radius of the event horizon which is the greater root of $f(r)=0$.

This can be reduced to the following simple equation,

$$
r^{2}-m-2 Q^{2} \ln (r)=0,
$$

which is $\alpha$ independent. It is remarkable to observe that $\alpha$ does not change the radius of the event horizon.

To go further, let us take $m=1$, with $Q<1$, then one can easily show that the particular radius of the event horizon $r_{+}$is equal to 1 and consequently

$$
\kappa=\frac{1-Q^{2}}{4 \alpha+1} \text {. }
$$

Clearly at the EYM limit (i.e., $\alpha \rightarrow 0$ ) $\kappa=1-Q^{2}$ and asymptotically when $\alpha \rightarrow \infty, \kappa \rightarrow 0$, which states that the space is flat. The associated Hawking temperature is given by

$$
T_{H}=\frac{\kappa}{2 \pi}=\frac{1}{2 \pi} \frac{1-Q^{2}}{4 \alpha+1}
$$

in natural units $c=G=\hbar=k=1$.

The expression (13) suggests that the square root term must be positive; this restricts our choice of the GaussBonnet parameter $\alpha$ to certain limits. In order to get rid of the negative sign in the square root for any arbitrary $\alpha$, one can shift the origin to the largest root of the square root term [4].

As a final remark we wish to express optimism that in a similar manner it is possible to construct analogous solutions for the EYMGB equations in higher dimensions. This all amounts to defining appropriate gauge potentials and overcoming the tedious calculations. 
[1] T. T. Wu and C. N. Yang, in Properties of Matter Under Unusual Conditions, edited by H. Mark and S. Fernbach (Interscience, New York, 1969), p. 349.

[2] P. B. Yasskin, Phys. Rev. D 12, 2212 (1975).

[3] C. W. Misner, K. S. Thorne, and J. A. Wheeler, Gravitation (Freemann, San Fransisco, 1973).
[4] M.H. Dehghani, Phys. Rev. D 70, 064019 (2004).

[5] N. Okuyama and K. Maeda, Phys. Rev. D 67, 104012 (2003).

[6] S. A. Ridgway and E. J. Weinberg, Phys. Rev. D 52, 3440 (1995). 